\title{
UNA ESPECIE NUEVA DE BEAUCARNEA (NOLINACEAE)
}

\author{
Luis Hernandez Sandoval' \\ Instituto de Ecología y Alimentos \\ Universidad Autónoma de Tamaulipas \\ Blvd. Lopez Mateos 928. Cd. Victoria 87040 \\ Tamaulipas, México
}

\section{RESUMEN}

Se describe Beaucarnea hiriartiae sp. $n$. del estado de Guerrero, México. Esta nueva especie es similar a B. stricta Lemaire de los estados vecinos de Puebla y Veracruz.

\section{ABSTRACT}

Beaucarnea hiriartiae sp. $\mathrm{n}$. is described from the state of Guerrero, Mexico. This new species is similar to B. stricta Lemaire from the neighbor states of Puebla and Veracruz.

Como parte del proyecto de tesis de postgrado del autor, se encontraron poblaciones de una especie del género Beaucarnea en la cuenca media del río Balsas, Guerrero. Las primeras colectas de esta planta datan de 1948 por Moore y Wood y de 1949 por Faustino Miranda. En las anotaciones de los especimenes correspondientes se observa que los mencionados botánicos la consideraron como una especie nueva. Sin embargo, en colectas recientes ha sido confundida con Beaucarnea stricta Lemaire. Al estudiar con detalle más ejemplares de herbario, así como las poblaciones naturales de esta planta, se concluyó que se trata de un nuevo taxon.

Beaucarnea hiriartiae L. Hernández S. sp. nov. (Fig. 1).

B. stricta Lemaire affinis, a qua differt basi caulis in sectione transversali elliptica, cortice plumbeo, foliis longioribus et recurvatis, inflorescentiis gracilioribus ramulis applanatis, bracteis inflorescientiae spiralibus vel ad basim plicatis, pedicellis fructus longioribus et fructu ad apicem non profunde emarginato.

Planta dioica, arborescente, de hasta $8 \mathrm{~m}$ de altura, esparcidamente ramificada, corteza lisa, gris brillante, formando placas, poligonales en la base y, cuadradas a

\footnotetext{
' Dirección actual: Department of Botany, University of Texas. Austin, Texas. 78713. U.S.A.
} 

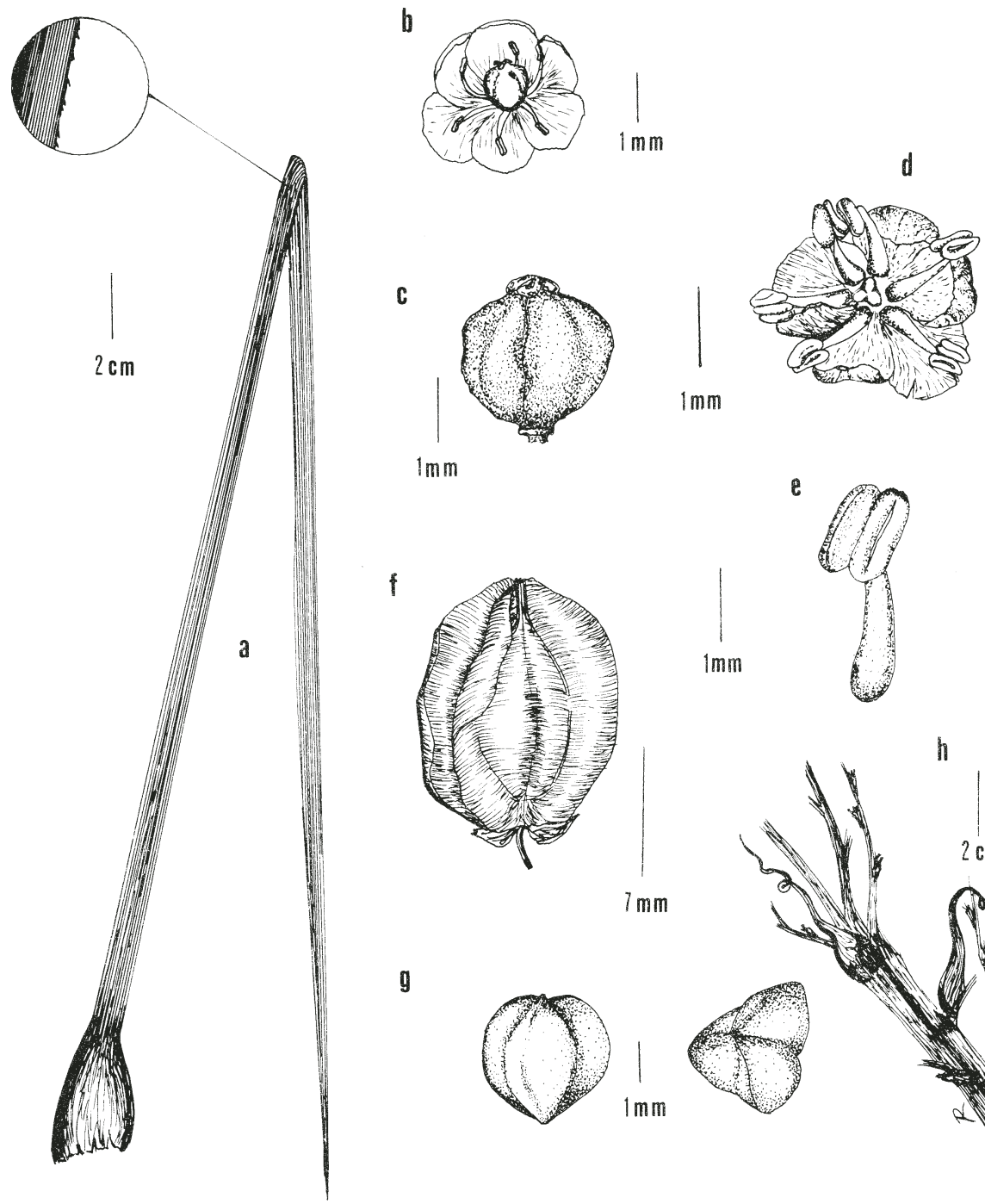

$$
\text { . }
$$
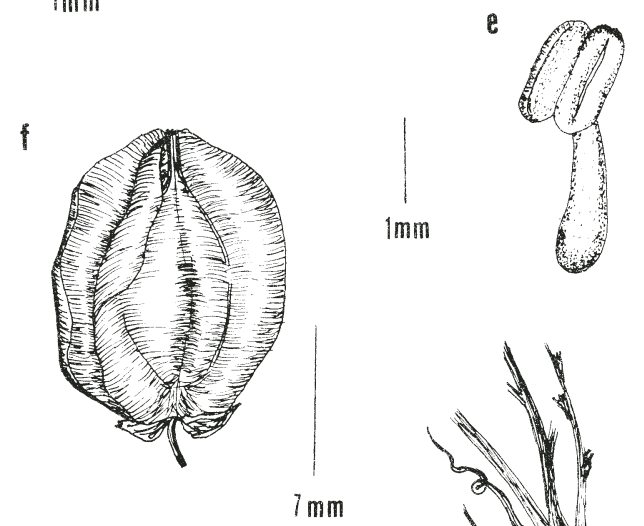

g
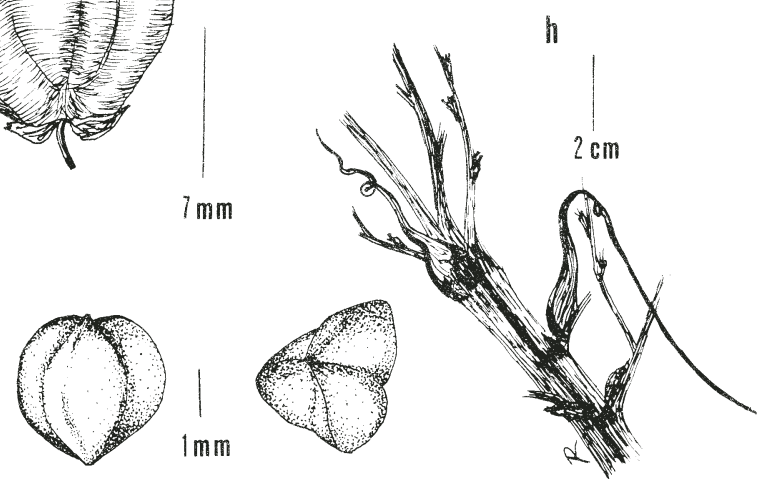

Fig. 1. Beaucarnea hiriartiae. a. Hoja joven con borde microserrulado; b. Flor femenina con estaminodios; c. Ovario fecundado; d. Flor masculina con ovario rudimentario; e. Estambre; f. Fruto; g. Semilla; h. Sección de inflorescencia mostrando brácteas en espiral. 
rectangulares en el tronco; base del tronco ensanchada, elíptica en sección transversal a manera de contrafuerte, de $1 \mathrm{~m}$ de alto, de $2 \mathrm{~m}$ de diámetro; ramas delgadas y largas, corteza grisácea brillante. Hojas lineares con la base deltoidea, de 2.5 a $4.5 \mathrm{~cm}$ de largo, 2 a $3 \mathrm{~cm}$ ancho en la parte inferior y 0.6 a $1.2 \mathrm{~cm}$ en la superior, blanca, amplexicaule, margen de 1 a $2 \mathrm{~mm}$ de ancho, membranoso y translúcido, microserrulado, dientecillos de 0.1 a $0.2 \mathrm{~mm}$ de largo; láminas recurvadas, de 70 a $90 \mathrm{~cm}$ de largo, 1 a $1.5 \mathrm{~cm}$ de ancho en la mitad de la hoja, de color verde pálido, cóncavas, con una ligera quilla, acanaladas, papiladas en los canales. Inflorescencia terminal, bracteada, paniculada, elipsoide a ovoide, de 0.7 a $1 \mathrm{~m}$ de alto; pedúnculo de 10 a $15 \mathrm{~cm}$ de largo, 1.2 a 1.5 $\mathrm{cm}$ de diámetro, brácteas largamente triangulares, de 40 a $50 \mathrm{~cm}$ de largo, $2.5 \mathrm{~cm}$ de ancho en la base, foliáceas, onduladas en la base, de color verde pálido a amarillentas; ramas de la inflorescencia planas, las inferiores de 20 a $25 \mathrm{~cm}$ de largo, brácteas subyacentes onduladas o en espiral, las ramas superiores y ramillas secundarias (en algunos casos se encuentran ramillas terciarias) de 3 a $10 \mathrm{~cm}$ de largo, brácteas subyacentes lanceolado-lineares' de 2 a $5 \mathrm{~cm}$ de largo, de forma helicoidal; fascículos florales con brácteas subyacentes lanceoladas de 3 a $3.5 \mathrm{~mm}$ de largo, membranosas; bractéolas envolviendo las yemas florales, obovadas en las estaminadas, lanceoladas en las pistiladas, de 2 a $3 \mathrm{~mm}$ de largo, 1 a $2 \mathrm{~mm}$ de ancho, ligeramente laciniadas con la edad. Flores estaminadas 2 a 3 por nudo, esferoidales, de 3 a $3.5 \mathrm{~mm}$ de diámetro; pedicelos de 2.5 a $3.5 \mathrm{~mm}$ de largo, articulados en su segundo tercio; segmentos del perianto 6 , de $3 \mathrm{~mm}$ de largo, $2 \mathrm{~mm}$ de ancho, ovales, cóncavos, blanco-amarillentos, margen ligeramente denticulado a subentero; estambres 6 , filiformes, filamentos de 2.5 a $3 \mathrm{~mm}$ de largo, anteras sagitadas, de 0.8 a $1 \mathrm{~mm}$ de largo, $0.4 \mathrm{~mm}$ de ancho, inserción del filamento entre la mitad y el tercio superior de la antera; ovario rudimentario, de 0.5 a $0.8 \mathrm{~mm}$ de largo. Flores pistiladas 2 por nudo, esferoidales, de 2.5 a $3 \mathrm{~mm}$ de diámetro, pedicelos 3.5 a $4.5 \mathrm{~mm}$ de largo, articulados en su segundo tercio; segmentos del perianto anchamente ovados, $2 \mathrm{~mm}$ de largo, 1.5 a $1.8 \mathrm{~mm}$ de ancho, cóncavos, blancoamarillentos; estaminodios 6 , de $2 \mathrm{~mm}$ de largo; ovario anchamente ovoide, de 2.5 a 3 $\mathrm{mm}$ de diámetro, con tres lobulaciones que se ensanchan en la parte superior; estilo subsésil, de $0.5 \mathrm{~mm}$ de largo, cortamente alado; estigma trilobado, densamente papilado; nectarios 3, septales. Frutos secos, trialados, obovoides a oblongos. de 0.8 a $1.1 \mathrm{~cm}$ de largo y 0.7 a $1 \mathrm{~cm}$ de ancho, amarillentos, ápice entero, truncado; pedúnculos de 5.5 a $6.5 \mathrm{~mm}$ de largo, articulados en su segundo tercio; el estilo llega a crecer hasta 1.50 $2 \mathrm{~mm}$ de largo en el fruto maduro. Semillas elipsoides a obovoides, de 3.5 a $4 \mathrm{~mm}$ de largo, 3 a $3.2 \mathrm{~mm}$ de ancho, pardo-rojizas, con tres lóbulos mucronulados, uno ligeramente mayor que los otros dos; embrión cilindrico, de 3 a $3.5 \mathrm{~mm}$ de largo, 0.7 a $0.9 \mathrm{~mm}$ de ancho.

TIPO: México, Guerrero: $12 \mathrm{~km}$ al sur de Mezcala; 3.I.1986; Hernández S. \& Martínez 1629, (holotipo MEXU; isotipos TEX, UAT).

Material adicional examinado: México, Guerrero. Cañón del Zopilote, just north of Venta Vieja, km 263 on highway between Chilpancingo and Mexcala, 23.VIII.1948, H. E. Moore \& C. E. Wood Jr. 4742 (GH, MICH); Cañada Zopilote, X.1949, F. Miranda 4313 (GH, MEXU, US); Cañón del Zopilote, $10 \mathrm{~km}$ al $\mathrm{N}$ de Zumpango, km 246 on Hwy 95 (17 $40^{\prime}$ lat. N, $99^{\circ} 30^{\prime}$ long. W), 10.IX.1965, Roe \& Roe 1925 (MICH, NY); $14.7 \mathrm{mi}$ 
N of PEMEX at N side of Chilpancingo, XII.1972, Dunn, Dziekanowski \& Bolingbroke 20509 (NY, US); $12 \mathrm{~km}$ al S de Mezcala, Cañón del Zopilote, 12.IV.1985, Hernández \& Martínez 1631, 1632 (MEXU, TEX, UAT); $12 \mathrm{~km}$ al S de Mezcala, 3.I.1986, Hernández \& Martínez 1630 (MEXU, TEX, UAT); $10 \mathrm{~km}$ al $\mathrm{N}$ de Tlapa, puente del río Tlapaneco, 3.I.1987, Hernández \& Martínez 2143 (MEXU, TEX, UAT); Cañon del Zopilote, 28.I.1989, Franco Gaona 15 (MEXU); 12 km al S de Mezcala, 21.VII.1990, Hernández, Martínez, Franco \& Santamarina 2463 (MEXU, TEX, UAT).

Habitat. Cañones de la cuenca media del río Balsas, en ladera de pendiente muy pronunciada sobre lutitas y calizas, en altitudes que van de los 250 a los $700 \mathrm{~m}$, con una precipitación anual de $\pm 750 \mathrm{~mm}$, asociada al bosque tropical caducifolio (Rzedowski, 1978), usualmente cerca de ríos, arroyos o en lugares protegidos.

Fenología. Presenta dos períodos de floración seguidos por otros dos de fructificación. Florece de diciembre a abril y de mayo a septiembre; fructifica de enero a mayo y de julio a octubre.

Nombres comunes. "Izote delgado", "ixtotl" (al parecer se conoce esta especie como una extensión del nombre náhuatl asignado a plantas del género Yucca en los alrededores de Tlapa).

Según la clasificación de Trelease (1911), Beaucarnea hiriartiae quedarla ubicada en la sección Papillatae (que incluye B. stricta y B. gracilis) por tener las hojas más o menos cóncavas, pálidas, con canales papilados y los frutos pequeños. Sin embargo, la nueva especie también presenta características de la sección Beaucarnea, tales como hojas delgadas, recurvadas y brácteas de los fascículos florales y pedicelos relativamente largos. Esta semejanza con B. recurvata Lemaire (1861) indica la necesidad de una revisión taxonómica del género. No obstante, se aprecia una mayor relación con las especies de la sección Papillatae, en especial con B. stricta. Beaucarnea hiriartiae es diferente de esta última por la corteza grisácea y brillante, además de las hojas delgadas, largas, recurvadas, el pedúnculo y las ramas de la inflorescencia más delgadas, las brácteas primarias y secundarias largas y enroscadas o plegadas por encima de la base y por sus pedicelos largos, eritre otras características (Cuadro 1). En cuanto al habitat, $B$. stricta ocupa zonas más secas (de 400 a $500 \mathrm{~mm}$ de precipitación anual), ubicadas a mayores altitudes (de 1000 a 2000 m s.n.m.), con menores pendientes y crece en matorrales xerófilos 0 en la transición de estas comunidades con bosques de Quercus (Rzedowski, 1978).

El nombre de esta especie se dedica a la eminente botánica y maestra Patricia Hiriart Valencia. 
Cuadro 1. Comparación morfológica entre las especies de la sección Papillatae propuesta por Trelease (1911) y Beaucarnea hiriartiae.

\begin{tabular}{|c|c|c|c|}
\hline & B. hiriartiae & B. stricta & B. gracilis \\
\hline Distribución & Guerrero & Puebla, Oaxaca & Puebla, Oaxaca \\
\hline $\begin{array}{l}\text { Base del tronco } \\
\text { secc. transv. }\end{array}$ & ovalada & circular & circular \\
\hline Hojas & recurvadas & erectas & erectas \\
\hline $\begin{array}{l}\text { Longitud de la } \\
\text { hoja }(\mathrm{cm})\end{array}$ & $70-90$ & $55-80$ & $30-60$ \\
\hline $\begin{array}{l}\text { Ancho a la mitad } \\
\text { de la hoja }(\mathrm{cm})\end{array}$ & $1-1.5$ & $0.8-1.5$ & $0.4-0.7$ \\
\hline $\begin{array}{l}\text { Diámetro en la base } \\
\text { del pedúnculo }(\mathrm{cm})\end{array}$ & $1.2-1.7$ & $2-4$ & $1.5 \cdot 1.8$ \\
\hline Brácteas & $\begin{array}{l}\text { onduladas y } \\
\text { en espiral }\end{array}$ & algo onduladas & no onduladas \\
\hline $\begin{array}{l}\text { Ramas de la } \\
\text { inflorescencia }\end{array}$ & aplanadas & $\begin{array}{l}\text { cilindricas } \\
\text { o anguladas }\end{array}$ & $\begin{array}{l}\text { cilíndricas } \\
\text { o anguladas }\end{array}$ \\
\hline $\begin{array}{l}\text { Longitud de pedicelos } \\
\text { en fruto }(\mathrm{mm})\end{array}$ & 5.5. -6.5 & $2.8 \cdot 3$ & $3.2-3.5$ \\
\hline $\begin{array}{l}\text { Largo } \times \text { ancho } \\
\text { de segmentos del } \\
\text { perianto }(\mathrm{mm})\end{array}$ & $2.5 \times 2$ & $2.5 \times 2$ & $1.2 \times 1$ \\
\hline $\begin{array}{l}\text { Largo } x \text { ancho de } \\
\text { semillas }(\mathrm{cm})\end{array}$ & $3.5 \times 3.1$ & $3.6 \times 3.3$ & $3.6 \times 2.8$ \\
\hline
\end{tabular}

\section{AGRADECIMIENTOS}

Se agradece a Fernando Chiang y a Guy Nesom la traducción al latín de la diagnosis, a Alfonso Delgado la revisión del manuscrito, a Rosalinda Medina la ilustración y a Esteban Martínez su valiosa ayuda.

\section{LITERATURA CITADA}

Lemaire, Ch. 1861. Genre nouveau de la famille Asparagacoés. Beaucarnea. Ilius. Hort. 8: 57-62.

Rzedowski, J. 1978. Vegetación de México. Ed. Limusa. México, D.F. pp. 189-204, 237-282.

Trelease, W. 1911. The desert group Nolineae. Proc. Amer. Phil. Soc. 50: 401-441. 